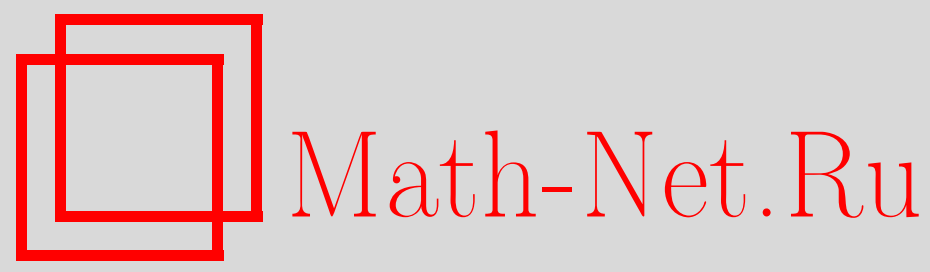

А. В. Субботин, Функциональные свойства пространств Привалова голоморфных функций нескольких переменных, Матем. заметки, 1999, том 65, выпуск 2, 280-288

DOI: https://doi.org/10.4213/mzm1050

Использование Общероссийского математического портала Math-Net.Ru подразумевает, что вы прочитали и согласны с пользовательским соглашением http://www.mathnet.ru/rus/agreement

Параметры загрузки:

IP : 3.89 .185 .249

26 апреля 2023 г., 16:56:17

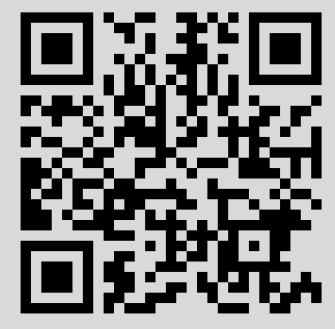


том 65 выпУСК 2 фЕВРАль 1999

УДК 517.53

\section{ФУНКЦИОНАЛЬНЫЕ СВОЙСТВА ПРОСТРАНСТВ ПРИВАЛОВА ГОЛОМОРФНЫХ ФУНКЦИЙ НЕСКОЛЬКИХ ПЕРЕМЕННЫХ}

\section{А. В. Субботин}

В единичном шаре и поликруге из $\mathbb{C}^{n}$ рассмотрены классы И. И. Привалова степеней $q>1$, определяемые как множество голоморфных, например в шаре, функций $f(z)$, у которых $\ln _{+}^{q}|f(z)|$ имеют ограниченные средние по сферам с центрами в нуле при возрастании радиусов к 1 . Классы (в одномерном случае) были введены Приваловым еще до 1941 года и в последние 10-20 лет часто возникали в иностранной литературе под разнообразными обозначениями, правда без упоминания о Привалове. В настоящей работе обсуждаются эквивалентные определения этих классов, а также свойства самого общего характера: оценки роста, свойства естественной метрики, ограниченность и полная ограниченность подмножеств.

Библиографоия: 13 названий.

1. Введение. Пусть $n$ - произвольное натуральное число и $\mathbb{C}^{n}-$ множество всех упорядоченных наборов $\left(z_{1}, \ldots, z_{n}\right)$ из $n$ комплексных чисел. Для $z, \zeta \in \mathbb{C}^{n}$ через $\langle z, \zeta\rangle$ будем обозначать эрмитово скалярное произведение $\sum_{k=1}^{n} z_{k} \bar{\zeta}_{k}$, через $|z|_{2}$ - порожденную им норму $\sqrt{\langle z, z\rangle}$ и через $|z|_{\infty}$ - норму, равную $\max _{1 \leqslant k} \leqslant n\left|z_{k}\right|$. Рассмотрим шар $G_{2}=\left\{z \in \mathbb{C}^{n}:|z|_{2}<1\right\}$ и поликруг $G_{\infty}=\left\{z \in \mathbb{C}^{n}:|z|_{\infty}<1\right\}$ и их граничные множества - сферу $\Gamma_{2}=\left\{\zeta \in \mathbb{C}^{n}:|\zeta|_{2}=1\right\}$ и остов поликруга $\Gamma_{\infty}=\left\{\zeta \in \mathbb{C}^{n}:\left|\zeta_{k}\right|=1,1 \leqslant\right.$ $k \leqslant n\},-$ а также комплексные ядра Пуассона для этих областей: для $z \in G_{2}$ и $\zeta \in \Gamma_{2}$

$$
P_{2}(z, \zeta)=\left(1-|z|_{2}^{2}\right)^{n}|1-\langle z, \zeta\rangle|^{-2 n}
$$

и для $z \in G_{\infty}$ и $\zeta \in \Gamma_{\infty}$

$$
P_{\infty}(z, \zeta)=\prod_{k=1}^{n}\left(1-\left|z_{k}\right|^{2}\right)\left|1-z_{k} \bar{\zeta}_{k}\right|^{-2}
$$

Стандартной мерой на сфере $\Gamma_{2}$ является нормированная площадь Лебега, а на торе $\Gamma_{\infty}$ - нормированное произведение мер Лебега единичных окружностей, составляющих тор. Обозначим их через $\sigma_{2}$ и $\sigma_{\infty}$ соответственно. Обсуждение свойств введенных объектов см. в $[1$, гл. 1-4], [2, гл. I, II]. По-возможности, будем объединять оба случая (шара и поликруга), вводя единые обозначения: $|z|=|z|_{2} \vee|z|_{\infty}, G=G_{2} \vee G_{\infty}, \Gamma=\Gamma_{2} \vee \Gamma_{\infty}$, $P=P_{2} \vee P_{\infty}$ и $\sigma=\sigma_{2} \vee \sigma_{\infty}$. 
Рассмотрим голоморфную в области $G$ функцию $f(z)$. Скажем, что $f(z)$ принадлежит классу Привалова $N^{q}$ степени $q>1$, если существует конечная постоянная $K=K(f)$ такая, что при всех $r, 0 \leqslant r<1$,

$$
\int_{\Gamma} \ln _{+}^{q}|f(r \gamma)| \sigma(d \gamma) \leqslant K
$$

Здесь $\ln _{+} a=\max (0, \ln a)$ при $a>0$ и $\ln _{+} 0=0$.

Обобщим это определение. Пусть $\varphi(t), t \geqslant 0,-$ произвольная неотрицательная, неубывающая, выпуклая вниз функция. Скажем, что $f(z)$ принадлежит классу $\varphi(N)$, если существует конечная постоянная $N=N(f)$ такая, что при всех $r, 0 \leqslant r<1$,

$$
\int_{\Gamma} \varphi\left(\ln _{+}|f(r \gamma)|\right) \sigma(d \gamma) \leqslant N
$$

Полагая $\varphi(t)$ равной $t$ и $e^{p t}, p>0$, соответственно получаем класс Неванлинны $N$ и классы Харди $H^{p}$ (см. [1, гл. 5, п. 5.6] и [2, гл. III, п. $\left.\left.3.3,3.4\right]\right)$. Случай $\varphi(t)=t^{q}, q>1$, соответствует классам Привалова $N^{q}$. В одномерном случае классы $N^{q}$ были рассмотрены в монографии И.И. Привалова [3], затем изучались в [4]-[6]. По поводу общих классов $\varphi(N)$ см. [7].

Несколько иной подход к определению классов дает радиальная максимальная функция

$$
M_{\mathrm{rad}} f(\gamma)=\sup _{0 \leqslant r<1}|f(r \gamma)|, \quad \gamma \in \Gamma .
$$

Скажем, что $f(z)$ принадлежит классу $\varphi(M)$, если

$$
\int_{\Gamma} \varphi\left(\ln _{+} M_{\mathrm{rad}} f(\gamma)\right) \sigma(d \gamma)<+\infty
$$

Значит, семейство функций на $\Gamma$

$$
\Psi_{r}(\gamma)=\varphi\left(\ln _{+}|f(r \gamma)|\right), \quad 0 \leqslant r<1,
$$

обладает интегрируемой мажорантой. При $\varphi(t)=t$ класс $M$ в одномерном случае был введен Х. О. Кимом в [8] и исследовался в [9], затем многомерные и степенные обобщения $M^{q}$ на случай $\varphi(t)=t^{q}, q>0$, и $n \geqslant 1$ изучались в [10], [11].

Подчиняя семейство (1) немного более слабому условию - равностепенной (по $r$ ) абсолютной непрерывности интегралов на $\Gamma$ - получаем условие принадлежности $f(z)$ к классу $\varphi\left(N_{*}\right)$. Здесь свойство равностепенной абсолютной непрерьвности означает, что для любого $\varepsilon>0$ найдется $\delta>0$ такое, что для любого $\sigma$-измеримого множества $E \subset \Gamma$ с мерой $\sigma E<\delta$ равномерно по $r, 0 \leqslant r<1$, вьполнено

$$
\int_{E} \Psi_{r}(\gamma) \sigma(d \gamma)<\varepsilon
$$

При $\varphi(t)=t$ получается известный класс Смирнова $N_{*}$ (см. [2, гл. III, п. 3.3]).

Включения $\varphi(M) \subseteq \varphi\left(N_{*}\right) \subseteq \varphi(N)$ очевидны. Классы $\varphi(N), \varphi\left(N_{*}\right)$ и $\varphi(M)$ не возрастают (по включению) при неубывании (по порядку роста на $+\infty) \varphi$. Следовательно, $H^{p} \subseteq N^{q} \subseteq N$ при $p>0, q>1$. Известно также, что $N_{*}=\cup \varphi(N)$, где объединение берется по всем растущим быстрее $t$ на $+\infty$ функциям $\varphi$ (см. [2, гл. III, теорема 3.1.2]). Учитьвая, что $\varphi(t)=t^{q}, q>1$, растет на $+\infty$ быстрее $t$, получаем уточнение последнего включения: $N^{q} \subseteq N_{*} \subseteq N$. Функции класса $N$ имеют почти всюду по мере $\sigma$ на $\Gamma$ радиальные граничные значения $\widetilde{f}(\gamma)=\lim _{r \uparrow 1} f(r \gamma)$ (см. [1, гл. 5, теорема 5.6.4], [2, гл. III, теорема 3.3.5]). 


\section{2. Свойства эквивалентные определению $N^{q}$.}

Tеорема 1. Пусть $f \in N u \tilde{f}(\gamma)=\lim _{r \uparrow 1} f(r \gamma)-$ ее радиальные граничные значения, существующие почти всюду на Г. При $q>1$ следующие свойства функиии $f(z)$ әквивалентны:

1) $f \in N^{q}$;

2) $f \in N_{*} u \ln _{+}^{q}|\widetilde{f}| \in L^{1}(\Gamma, \sigma)$;

3) $\ln _{+}^{q}|\widetilde{f}| \in L^{1}(\Gamma, \sigma)$ и для любого $z \in G$

$$
\ln _{+}^{q}|f(z)| \leqslant \int_{\Gamma} P(z, \gamma) \ln _{+}^{q}|\widetilde{f}(\gamma)| \sigma(d \gamma)
$$

4) $f \in M^{q}$;

5) при всех $r, 0 \leqslant r<1$,

$$
\int_{\Gamma} \ln _{+}^{q}|f(r \gamma)| \sigma(d \gamma) \leqslant \int_{\Gamma} \ln _{+}^{q}|\widetilde{f}(\gamma)| \sigma(d \gamma)<+\infty
$$

6) выполнено

$$
\lim _{r \uparrow 1} \int_{\Gamma} \ln _{+}^{q}|f(r \gamma)| \sigma(d \gamma)=\int_{\Gamma} \ln _{+}^{q}|\widetilde{f}(\gamma)| \sigma(d \gamma)<+\infty
$$

7) $f \in N_{*}^{q}=\left(N_{*}\right)^{q}$.

В одномерном случае эквивалентность свойств 1), 6) и 7) была проверена еще в [3]. Для доказательства теоремы в общем случае нам понадобится следующее вспомогательное утверждение.

ЛЕмма 1. Пусть $X$ - измеримое пространство с неотрицательной конечной мерой $\mu$ и $\left(f_{n}(x)\right)$ - последовательность неотрицательных интегрируемых функиий, сходящаяся почти всюду по мере $\mu \kappa$ интегрируемой функиии $f(x)$. Равенство

$$
\lim _{n \rightarrow \infty} \int_{X} f_{n}(x) \mu(d x)=\int_{X} f(x) \mu(d x)
$$

справедливо тогда и только тогда, когда последовательность $\left(f_{n}\right)$ имеет равностепенно абсолютно непрерывные интегралы на $X$.

ДоКАЗАТЕЛЬСтво этой леммы для $X=[a, b]$ с обычной мерой Лебега см. в $[12$, разд. I, $\S 3$, п. 3.2]. Оно без труда обобщается на произвольньй случай.

ДоКАЗАТЕЛЬСТво тЕОРЕМЫ 1. Эквивалентность свойств 4), 1) и 2) получена в [10]. В работе [7, лемма 2 и теорема 3] показано, что из свойства 1) следуют 3), 5) и 6). Из свойств 5) и 6) легко следует свойство 1). Лемма 1 влечет эквивалентность свойств 6) и 7). Для полноты доказательства остается лишш показать импликацию 3$) \Longrightarrow 5$ ). Полагая в $(2) z=r \zeta(0 \leqslant r<1)$ и интегрируя по $\zeta$, имеем

$$
\int_{\Gamma} \ln _{+}^{q}|f(r \zeta)| \sigma(d \zeta) \leqslant \int_{\Gamma} \int_{\Gamma} P(r \zeta, \gamma) \sigma(d \zeta) \ln _{+}^{q}|\widetilde{f}(\gamma)| \sigma(d \gamma)
$$


где порядок интегрирования изменен (по теореме Фубини). Используя свойства симметрии $P(r \zeta, \gamma)=P(r \gamma, \zeta)$ и нормированности (см. [1, гл. 3, предложение 3.3.3], [2, гл. II, п. 2.1])

$$
\int_{\Gamma} P(z, \gamma) \sigma(d \gamma)=1, \quad z \in G,
$$

ядра Пуассона, получаем требуемое неравенство (3).

ЗАмечаниЕ 1. Теорема 1 , исключая быть может лиш свойство 4), сохраняет свою силу и при замене $\varphi(t)=t^{q}$ на произвольную растущую сильнее $t$ на $+\infty$ функцию $\varphi$ (неотрищательную, неубьвающую и вьпуклую вниз). Следовательно, $\varphi(N)=\varphi\left(N_{*}\right)$ для таких $\varphi$. Будут ли при этом совпадать $\varphi(N)$ и $\varphi(M)$, большой вопрос. Используя одну интерполяционную теорему Зигмунда (см. [1, гл. 5, теорема 5.7]), можно показать лишь то, что $N \ln _{+} N \subset M$.

3. Оценка роста функций из $N^{q}$. Пусть $f(z)$ голоморфна в $G$. Функция $\ln ^{q}(1+$ $|f(z)|)$ так же, как и $\ln _{+}^{q}|f(z)|$ плюрисубгармонична в области $G$ для всякой голоморфной там функции $f(z)$ и всякого $q \geqslant 1$ (т.е. субгармонична при подстановке любого голоморфного отображения из единичного круга в $G)$. Действительно, $\ln ^{q}(1+|f(z)|)$ плюрисубгармонична как композиция плюрисубгармоничной $\ln |f(z)|$ и неубывающих, выпуклых вниз функций $\psi(s)=\ln (1+\exp s)$ и $\varphi(t)=t^{q}$. Поэтому теорема 1 сохраняет свою силу при замене в ней $\ln _{+}^{q}|f(z)|$ на $\ln ^{q}(1+|f(z)|)$.

Согласно лемме 2 из [7] интеграл

$$
I(r, f)=\int_{\Gamma} \ln ^{q}(1+|f(r \gamma)|) \sigma(d \gamma)
$$

не убывает по $r$ при $0 \leqslant r<1$ для любой голоморфной в $G$ функции $f(z)$. Поэтому $\lim _{r \uparrow 1} I(r, f)^{1 / q}$ существует всегда, конечньй для функций из $N^{q}$ и бесконечньй для остальных. Обозначим эту числовую характеристику через $|f|_{N}$. Согласно свойству 6) теоремы 1 (с заменой $\ln _{+}^{q}|f(z)|$ на $\left.\ln ^{q}(1+|f(z)|)\right)$ при $q>1$ (при $q=1$ это неверно)

$$
|f|_{N^{q}}=\left(\int_{\Gamma} \ln ^{q}(1+|\tilde{f}(\gamma)|) \sigma(d \gamma)\right)^{1 / q}
$$

где $\widetilde{f}(\gamma)=\lim _{r \uparrow 1} f(r \gamma)$ - радиальные граничные значения функции $f(z)$.

Используя неравенство

$$
P(z, \zeta) \leqslant(1+|z|)^{n}(1-|z|)^{-n}, \quad z \in G, \quad \zeta \in \Gamma
$$

и свойство 3$)$ теоремы $1\left(\ln ^{q}(1+|f(z)|)\right)$, можно получить простейшую оценку функций из $N^{q}$ :

$$
\ln (1+|f(z)|) \leqslant\left(\frac{1+|z|}{1-|z|}\right)^{n / q}|f|_{N^{q}}
$$

Однако, свойство 3) позволяет доказать более точную оценку роста. 
Teорема 2. Пусть $q>1 u f \in N^{q}$. Тогда для всякого $\varepsilon>0$ найдется $A=$ $A(\varepsilon)<+\infty$ maкая, что

$$
\ln _{+}|f(z)| \leqslant A+\frac{\varepsilon}{(1-|z|)^{n / q}}
$$

m.e. $\ln _{+}|f(z)|=o\left((1-|z|)^{-n / q}\right)$ npu $|z| \uparrow 1$.

ДокАЗАТЕльСтво. Пусть дано $\varepsilon>0$. Согласно абсолютной непрерьвности интеграла от $\ln _{+}^{q}|\widetilde{f}|$ найдется $\delta>0$ такое, что для любого $\sigma$-измеримого множества $E \subseteq \Gamma$ с $\sigma E<\delta$ справедливо

$$
\int_{E} \ln _{+}^{q}|\widetilde{f}(\gamma)| \sigma(d \gamma)<\frac{\varepsilon^{q}}{2^{n}} .
$$

Пусть $k \in \mathbb{N}$ и $E_{k}=\left\{\gamma \in \Gamma: \ln _{+}|\widetilde{f}(\gamma)| \geqslant k\right\}$. Согласно неравенству Чебьшева

$$
\sigma E_{k} \leqslant \frac{1}{k^{q}} \int_{\Gamma} \ln _{+}^{q}|\widetilde{f}(\gamma)| \sigma(d \gamma) \leqslant \frac{|f|_{N^{q}}^{q}}{k^{q}} .
$$

Поэтому найдется $k \in \mathbb{N}$ такое, что $\sigma E_{k}<\delta$ (например, $k>|f|_{N^{q}} / \delta^{1 / q}$ ), значит для $E=E_{k}$ выполнено (8). Отсюда согласно свойству 3 ) теоремы 1 имеем

$$
\begin{aligned}
\ln _{+}|f(z)| & \leqslant\left(\int_{E_{k}} P(z, \gamma) \ln _{+}^{q}|\widetilde{f}(\gamma)| \sigma(d \gamma)\right)^{1 / q}+\left(\int_{\Gamma \backslash E_{k}} P(z, \gamma) \ln _{+}^{q}|\widetilde{f}(\gamma)| \sigma(d \gamma)\right)^{1 / q} \\
& \leqslant\left(\frac{1+|z|}{1-|z|}\right)^{n / q}\left(\int_{E_{k}} \ln _{+}^{q}|\widetilde{f}(\gamma)| \sigma(d \gamma)\right)^{1 / q}+k\left(\int_{\Gamma \backslash E_{k}} P(z, \gamma) \sigma(d \gamma)\right)^{1 / q} \\
& \leqslant \frac{\varepsilon}{(1-|z|)^{n / q}}+k
\end{aligned}
$$

мы воспользовались оценкой ядра Пуассона (6), неравенствами $(4),(8)$ и

$$
(a+b)^{1 / q} \leqslant a^{1 / q}+b^{1 / q}, \quad a, b \geqslant 0 .
$$

Следовательно, теорема доказана с $A(\varepsilon)=k$.

4. $N^{q}$ как $F$-алгебра. Пусть $f(z), g(z)$ - голоморфные в $G$ функции и $q \geqslant 1$. Из простых неравенств

$$
\begin{gathered}
\ln (1+a+b) \leqslant \ln (1+a)+\ln (1+b), \quad \ln (1+a b) \leqslant \ln (1+a)+\ln (1+b), \\
\min (1, \alpha) \ln (1+a) \leqslant \ln (1+\alpha a) \leqslant \max (1, \alpha) \ln (1+a),
\end{gathered}
$$

справедливых для $a, b, \alpha \geqslant 0$, и неравенства Минковского в $L^{q}(\Gamma, \sigma)$ при $q \geqslant 1$ можно вывести простейшие свойства характеристики $|\cdot|_{N^{q}}$, введенной в п. 3 :

$$
\begin{gathered}
|f+g|_{N^{q}} \leqslant|f|_{N^{q}}+|g|_{N^{q}}, \quad|f g|_{N^{q}} \leqslant|f|_{N^{q}}+|g|_{N^{q}}, \\
\min (1,|\alpha|)|f|_{N^{q}} \leqslant|\alpha f|_{N^{q}} \leqslant \max (1,|\alpha|)|f|_{N^{q}},
\end{gathered}
$$

где $\alpha \in \mathbb{C}$. Отсюда автоматически следует замкнутость $N^{q}, q \geqslant 1$, относительно поточечного сложения и умножения, т.е. $N^{q}$ - коммутативная алгебра. Характеристика $|\cdot|_{N^{q}}$ обладает на $N^{q}$ тремя свойствами:

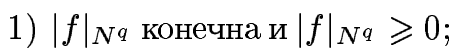

2) из $|f|_{N^{q}}=0$ следует, что $f \equiv 0$ в $G$;

3) $|f+g|_{N^{q}} \leqslant|f|_{N^{q}}+|g|_{N^{q}}$. 
Характеристики с такими свойствами называются квазинормами. Каждая квазинорма на линейном пространстве порождает каноническим образом инвариантную относительно сдвигов метрику: $\rho_{N^{q}}(f, g)=|f-g|_{N^{q}}$ (для $\left.f, g \in N^{q}\right)$. Свойства этой метрики и будут занимать наше внимание.

Аналогично первому утверждению теоремы 4.2 из [4] имеет место следующая теорема (многомерность не играет существенной роли).

Теорема 3. При $q>1$ метрика $\rho_{N^{q}}$ превращает $N^{q}$ в $F$-алгебру относительно поточечного слохения и умножения, т.е. метрика инвариантна при сдвигах, полна и операчия умножения непрерывна в әтой метрике, причем

$$
|f g|_{N^{q}} \leqslant|f|_{N^{q}}+|g|_{N^{q}} .
$$

При $q=1$ утверждение теоремы становится неверньм (см. [13]). Другому утверждению теоремы 4.2 из [4] соответствует

Tеорема 4. Ecлu $q>1, f \in N^{q} u f_{r}(z)=f(r z), z \in G, 0 \leqslant r<1$, mo $f_{r} \rightarrow f n p u$ $r \uparrow 1$ nо метрике $N^{q}$.

Так как растяжения $f_{r}$ при $0 \leqslant r<1$ голоморфны на самом деле в области $G / r$, то в $G$ они представимы равномерно сходящимся рядом Тейлора. Поэтому из теоремы 3 непосредственно вытекает

СледСТВИЕ. Многочлены плотны в $N^{q}$ при $q>1$ и $N^{q}$ - сепарабельная $F$-алгебра.

В [4] М. Столл показал (в одномерном случае), что функции, для которых справедлива оценка (7) (теорема 2), образуют алгебру $\Phi$ реше $F_{1 / q}$, накрьвающую $F$-алгебру $N^{q}$, и обстоятельно изучил свойства этой алгебры.

5. Ограниченные подмножества $N^{q}$. Согласно теореме 3 пространства $N^{q}$ при $q>1$ являются линейными топологическими пространствами, т.е. операции сложения и умножения на скаляр непрерьвны в метрике $\rho_{N} q$. Подмножество $B$ линейного топологического пространства называется ограниченным, если для любой окрестности нуля $U$ существует $\alpha_{0}>0$ такое, что для всех скаляров $\alpha,|\alpha| \leqslant \alpha_{0}$, имеет место включение $\alpha B \subset U$. В случае, когда топология линейного топологического пространства порождена метрикой, ограниченность в смысле линейного топологического пространства не всегда равносильна ограниченности попарных расстояний.

ТЕОРема 5. Ограниченность подмножества $L$ пространства $N^{q}, q>1, p a в-$ носильна выполнению следующих двух условий:

1) существует $K<+\infty$ такое, что $|f|_{N^{q}} \leqslant K$ для всех $f \in L$;

2) семейство $\left\{\ln _{+}^{q}|\widetilde{f}|\right\}_{f \in L}$ имеет равностепенно абсолютно непрерывные интегралы на Г.

ДокАЗАТЕЛЬСтво. Необходимость. Пусть $L$ - ограниченное в $N^{q}$ подмножество функций.

1) Взяв в определении ограниченности $U=\left\{f \in N^{q}:|f|_{N^{q}}<1\right\}$, видим, что при достаточно малом $\alpha_{0}>0$ квазинормы $\left|\alpha_{0} f\right|_{N^{q}}$ ограничены 1 при $f \in L$. Следовательно, в силу неравенства (11) ограничены при $f \in L$ и

$$
|f|_{N^{q}} \leqslant \max \left(1, \frac{1}{\alpha_{0}}\right)\left|\alpha_{0} f\right|_{N^{q}}<\max \left(1, \frac{1}{\alpha_{0}}\right) .
$$


Тем самьм, необходимость условия 1) установлена.

2) Докажем теперь равностепенную абсолютную непрерьвность интегралов семейства $\left\{\ln _{+}^{q}|\widetilde{f}|\right\}_{f \in L}$. А значит, для произвольно малого $\varepsilon>0$ необходимо указать $\delta>0$ такое, что для всех $\sigma$-измеримых множеств $E \subset \Gamma$ с мерой $\sigma E<\delta$ и любой функции $f \in L$ вьполнено

$$
\int_{\Gamma} \ln _{+}^{q}|\widetilde{f}(\gamma)| \sigma(d \gamma)<\varepsilon
$$

В силу неравенства $\ln _{+}^{q} t \leqslant \ln ^{q}(1+t), t \geqslant 0$, достаточно то же самое доказать для $\ln ^{q}(1+|\tilde{f}(\gamma)|)$ вместо $\ln _{+}^{q}|\tilde{f}(\gamma)|$.

Итак, пусть $\varepsilon>0$ произвольно мало. Так как $L$ ограничено, то для $U=\left\{f \in N^{q}\right.$ : $\left.|f|_{N^{q}}<\varepsilon^{1 / q} / 2\right\}$ найдется $\alpha_{0}>0$ такое, что $\alpha_{0} L \subset U$, т.е. для любой $f \in L\left|\alpha_{0} f\right|_{N^{q}}<$ $\varepsilon^{1 / q} / 2$. Зафиксировав это $\alpha_{0}$ и $f \in L$, для произвольного $\sigma$-измеримого множества $E \subset$ Г имеем

$$
\begin{aligned}
\left(\int_{E} \ln ^{q}(1+|\widetilde{f}(\gamma)|) \sigma(d \gamma)\right)^{1 / q} \leqslant & \left(\int_{E} \ln ^{q}\left(1+\left|\alpha_{0} \widetilde{f}(\gamma)\right|\right) \sigma(d \gamma)\right)^{1 / q} \\
& +\left(\int_{E} \ln ^{q}\left(1+\frac{1}{\alpha_{0}}\right) \sigma(d \gamma)\right)^{1 / q} \\
\leqslant & \left|\alpha_{0} f\right|_{N^{q}}+\ln \left(1+\frac{1}{\alpha_{0}}\right) \sigma E^{1 / q}
\end{aligned}
$$

мы воспользовались неравенством (10) (с заменой $f$ на $\alpha_{0} f, g$ на $1 / \alpha_{0}$ и $\Gamma$ на $E$ ) и выражением (5). Наконец, если выразим $\delta>0$ из равенства $\ln \left(1+1 / \alpha_{0}\right) \delta^{1 / q}=\varepsilon^{1 / q} / 2$, то при $\sigma E<\delta$ после небольших преобразований неравенства (12) получим

$$
\int_{E} \ln ^{q}(1+|\widetilde{f}(\gamma)|) \sigma(d \gamma)<\varepsilon
$$

равномерно по $f \in L$. Таким образом, необходимость условия 2 ) установлена.

Достаточность. Пусть теперь выполнены условия 1), 2). Чтобы показать ограниченность $L$, возьмем любую окрестность нуля $U$. Вписав в $U$ шар достаточно малого радиуса $\varepsilon>0$, без ограничения обшности можем считать $U=\left\{f \in N^{q}:|f|_{N^{q}}<\varepsilon\right\}$. В этом случае требуется показать, что при всех достаточно малых положительных по модулю $\alpha \in \mathbb{C} \alpha L \subset U$, т.е. $|\alpha f|_{N^{q}}<\varepsilon$ при всех $f \in L$. Для произвольных $\alpha \in \mathbb{C}$, $f \in N^{q}$ и $\sigma$-измеримого множества $E \subset \Gamma$ имеем

$$
\begin{aligned}
|\alpha f|_{N^{q}} & \leqslant \max (1,|\alpha|)\left(\int_{E} \ln ^{q}(1+|\tilde{f}(\gamma)|) \sigma(d \gamma)\right)^{1 / q}+\left(\int_{\Gamma \backslash E} \ln ^{q}(1+|\alpha \tilde{f}(\gamma)|) \sigma(d \gamma)\right)^{1 / q} \\
& =\max (1,|\alpha|) I_{1}+I_{2}
\end{aligned}
$$

мы воспользовались неравенствами (9) и (11) (с $E$ вместо Г). Так как

$$
\ln ^{q}(1+t) \leqslant 2^{q-1}\left(\ln ^{q} 2+\ln _{+}^{q} t\right), \quad t \geqslant 0,
$$

согласно условию теоремы для нашего $\varepsilon>0$ найдется $\delta>0$ такое, что при $\sigma E<\delta$ и $f \in L I_{1}<\varepsilon / 2$. Зафиксировав это $\delta>0$ и взяв $K$ из условия 1$)$, можно найти $m \in \mathbb{N}$ 
такое, что при всех $f \in L$ все множества $E_{m}=\{\gamma \in \Gamma:|\widetilde{f}(\gamma)| \geqslant m\}$ имеют меру $\sigma E_{m}<\delta$. Действительно, в силу неравенства Чебышева

$$
\sigma E_{m} \leqslant \frac{1}{\ln ^{q}(1+m)} \int_{\Gamma} \ln ^{q}(1+|\tilde{f}(\gamma)|) \sigma(d \gamma) \leqslant \frac{K^{q}}{\ln ^{q}(1+m)},
$$

значит достаточно выбрать $m>m_{0}$, где $m_{0}$ определяется из уравнения $K^{q} / \ln ^{q}\left(1+m_{0}\right)$ $=\delta$ (для $K=0 m$ можно выбрать любым). Итак, для $f \in L \sigma E_{m}<\delta$. Полагая $E=E_{m}$ в неравенстве (13) и вспоминая определения $\delta$ и $E_{m}$, имеем

$$
|\alpha f|_{N^{q}} \leqslant \max (1,|\alpha|) \frac{\varepsilon}{2}+\ln (1+|\alpha| m) ;
$$

откуда видно, что при достаточно малых (но не равных 0) по модулю числах $\alpha|\alpha f|_{N^{q}}<\varepsilon$ равномерно ( $m$ не зависит от конкретного выбора $f$ из $L !)$ по $f \in L$. Значит, $\alpha L \subset U$ при всех достаточно мальх по модулю ненулевых $\alpha$ и условие ограниченности для данной окрестности $U$ проверено.

Теорема доказана.

Приведем стандартный пример ограниченного в $N^{q}$ множества. Пусть $f \in N^{q}$ и $f_{r}(z)=f(r z), z \in G, 0 \leqslant r<1$. Тогда функции $f_{r}, 0 \leqslant r<1$, образуют ограниченное в $N^{q}$ подмножество. Условие 1) теоремы 5 вьполнено почти по определению принадлежности $f$ к $N^{q}$, а условие 2) есть не что иное, как свойство 7) теоремы 1.

6. Полная ограниченность подмножеств $N^{q}$. Как известно, полная ограниченность подмножеств полного метрического пространства эквивалентна их относительной компактности.

Теорема 6. Пусть $q>1$. Мнохсество $L \subset N^{q}$ вполне ограничено тогда и только тогда, когда

1) L ограничено в $N^{q}$ (в смысле линейного топологического пространства);

2) радиальные граничные значения функиий из L образуют относительно компактное по мере б подмножество измеримых функиий.

ДокАЗАТЕЛЬСтво. Необходимость. Множество $L$ ограничено в $N^{q}$ как любоевполне ограниченное подмножество линейного топологического пространства.

Покажем теперь, что множество $\widetilde{L}$ радиальных граничных значений функций из $L$ относительно компактно по мере. Пусть $\left(g_{n}\right) \subset \widetilde{L}-$ любая последовательность. Пусть $\widetilde{f}_{n}=g_{n}$, где $\left(f_{n}\right) \subset L$. Так как $L$ относительно компактно, то существует последовательность номеров $\left(n_{k}\right) \uparrow \uparrow \mathbb{N}, k \in \mathbb{N}$, такая, что $f_{n_{k}} \rightarrow f \in N^{q}$ при $k \rightarrow \infty$. Так как из сходимости в $N^{q}$ следует сходимость по мере радиальных граничных значений (см. неравенство (14)), то $g_{n_{k}} \rightarrow g=\widetilde{f}$ и по мере $\sigma$. Итак, из любой последовательности $\widetilde{L}$ мы выбрали сходящуюся по мере подпоследовательность. Значит, $\widetilde{L}$ относительно компактно.

Достаточность. Пусть $\left(f_{n}\right) \subset L$ - любая последовательность. Так как $\left(\widetilde{f}_{n}\right) \subset \widetilde{L}$, существует подпоследовательность $\tilde{f}_{n_{k}} \rightarrow g$ по мере $\sigma$ для некоторой измеримой $g$. По теореме Рисса из нееможно выделить сходящуюся почти всюду подпоследовательность, т.е. $f_{n_{k_{l}}} \rightarrow g$ при $l \rightarrow \infty$ почти всюду. Так как $L$ ограничено, по критерию ограниченности (см. теорему 5) последовательность $\ln ^{q}\left(1+\left|\widetilde{f}_{n_{k_{l}}}\right|\right)$ имеет ограниченные и равностепенно абсолютно непрерьвные интегралы на $\Gamma$. По теореме Фату функция $\ln ^{q}(1+|g|)$ 
интегрируема и, следовательно, последовательность $\ln ^{q}\left(1+\left|\widetilde{f}_{n_{k_{l}}}-g\right|\right)$ также имеет равностепенно абсолютно непрерьвные интегралы. Согласно лемме 1

$$
\lim _{l \rightarrow \infty} \int_{\Gamma} \ln ^{q}\left(1+\left|\tilde{f}_{n_{k_{l}}}-g\right|\right) d \sigma=0 .
$$

Отсюда следует, что $\left(f_{n_{k_{l}}}\right)$ фундаментальна в $N^{q}$, значит сходится. Итак, из всякой последовательности $L$ можно выделить сходящуюся подпоследовательность. Таким образом, $L$ относительно компактно.

Теорема доказана.

ЗАмечание 2. Пример ограниченного в $N^{q}$ множества, приведенный в конце п. 5, подходит и для примера вполне ограниченного множества. Но здесь относительная компактность этого множества очевидна. Действительно, любая последовательность здесь задается последовательностью $\left(r_{n}\right), 0 \leqslant r_{n}<1$. Если эта последовательность имеет предельную точку, отличную от 1 , то, выбрав соответствующую подпоследовательность $\left(r_{n_{k}}\right)$, сходящуюся к этой предельной точке, и взяв соответствующую подпоследовательность $\left(f_{r_{n_{k}}}\right)$ последовательности $\left(f_{r_{n}}\right)$, видим, что она сходится равномерно (к функции с номером, равньп предельной точке), а значит, и в метрике $N^{q}$. Если же $r_{n} \rightarrow 1$, то $f_{r_{n}} \rightarrow f$ по теореме 4 . Таким образом, из любой последовательности мы выделили сходящуюся подпоследовательность, т.е. относительная компактность имеет место.

\section{СПИСОК ЦИТИРОВАННОЙ ЛИТЕРАТУРЫ}

[1] Рудин У. Теория функций в шаре из $\mathbb{C}^{n}$. М.: Мир, 1984.

[2] Рудин У. Теория функций в поликруге. М.: Мир, 1974.

[3] Привалов И.И. Граничные свойства однозначных аналитических функций. М.: Изд-во МГУ, 1941.

[4] Stoll M. Mean growth and Taylor coefficients of some topological algebras of analytic functions // Ann. Polon. Math. 1977. V. 35. P. 139-158.

[5] Mochizuki N. Algebras of holomorphic functions between $H^{p}$ and $N_{*} / /$ Proc. Amer. Math. Soc. 1989. V. 105. P. 898-902.

[6] Meštrović R., Pavićević Ž. Remarks on some classes of holomorphic functions // Math. Montisnigri. 1996. V. 6. P. 27.

[7] Stoll M. Harmonic majorants for plurisubharmonic functions // J. Reine Angew. Math. 1976. V. 282. P. $80-87$.

[8] Kim H. O. On closed maximal ideals of $M$ // Proc. Japan Acad. Ser. A. Math. Sci. 1986. V. 62. № 9. P. 343-346.

[9] Kim H. O. On an F-algebra of holomorphic functions // Canad. J. Math. 1988. V. 40. № 3 . P. 718-741.

[10] Choe B. R., Kim H. O. On the boundary behavior of functions holomorphic on the ball // Complex Variables Theory Appl. 1992. V. 20. P. 53-61.

[11] Kim H. O., Park Y. Y. Maximal functions of plurisubharmonic functions // Tsukuba J. Math. 1992. V. 16. №1. P. 11-18.

[12] Привалов И. И. Граничные свойства аналитических функций. 2-е изд. М.-Л.: ГИТТЛ, 1950.

[13] Shapiro J., Shields A. Unusual topological properties of the Nevanlinna class // Amer. J. Math. 1975. V. 97. P. 915-936. 